\title{
EL EMPRENDIMIENTO EN EL MARCO DE LA GOBERNANZA
}

\section{ENTREPRENEURSHIP IN THE FRAMEWORK OF GOVERNANCE}

\section{Autor 1}

María Angélica Barrera Sánchez

Técnica en Nómina y Prestaciones Sociales y Tecnóloga en Gestión de Talento Humano del SENA y estudiante del programa de Economía de Escuela de Ciencias Administrativas, Contables, Económicas y de Negocios. Universidad Nacional Abierta y a Distancia UNAD, Bogotá.

ORCID: https://orcid.org/0000-0003-2331-8479

Correo electrónico: mabarreras@unadvirtual.edu.co

\section{Resumen}

El sistema económico, político y social también ha venido sufriendo cambios constantes, por lo tanto se han visto en la obligación de evolucionar a la par con la globalización, a raíz de esto la gobernanza busca hacer frente a los problemas que surgen en la sociedad, partiendo del diseño y desarrollo de las políticas públicas para promover los emprendimientos en los que tanto los actores públicos como los privados pueden hacerse partícipes para dar una respuesta de manera equitativa a las necesidades actuales.

Uno de los problemas que se aborda es que la economía presenta debilidades y que estas deben ser mitigadas por los actores políticos para salvaguardar el empleo, el crecimiento y el desarrollo económico a partir de los emprendimientos, sin embargo, debe involucrarse los actores sociales es decir los ciudadanos mediante mecanismos de participación.

La finalidad del presente escrito es brindar las definiciones, características y establecer la relación entre la gobernanza, el emprendimiento y las políticas públicas como generadoras de crecimiento y desarrollo en pro de dar respuesta a las necesidades actuales.

Palabras clave: gobernanza, política pública, economía, crecimiento, emprendimiento.

\section{Abstract}

The economic system, politic and social has also been suffering constantly changing, therefore have been in the obligation progress with the globalization, following this the governance search to make address issues that issue in the society, based in the design and development of the public 
politics for to promote the entrepreneurships in where the publics and privates actors can be participate for to give an answer is equitable to current necessities.

One of the problems that address is that the economy present weakness and that this to be alleviated for the politics actors for protect the employment, the growth and development economic from that the entrepreneurship, however should be involved the social actors is to say the civic by participation mechanism.

The document purpose of the present is to give the definitions, characteristics and to establish the relation among the governance, the entrepreneurship and the publics politics as generate of the growth and development for to give answer the problems.

Keywords: Governance, Public Politics, Economy, Growth, Entrepreneurship.

\section{INTRODUCCIÓN}

Con globalización, todos los ámbitos deben evolucionar, por lo tanto, el gobierno se ha visto en la necesidad de establecer una relación entre ciudadanos y gobierno que permita un manejo de las situaciones y que dé respuesta a los problemas que enfrenta la sociedad, en este sentido la gobernanza comienza a jugar un papel fundamental en la sociedad. (Jorquera, D. 2011). Por otro lado, Aguilar, F. (2010) aborda el término de gobernanza como punto referente en las transformaciones que ha tenido el gobierno y que con esto el modo de dirigir ha cambiado.

Los autores Calvo, G. Pérez, H. Romero M. (2020) nos hablan de que la gobernanza reúne actores globales de diferente naturaleza, pues según Jorquera, D. (2011) es un modelo que plantea mejores estrategias y políticas para resolver los problemas. Calvo, G. Pérez, H. Romero M. (2020) establecen que estas estrategias se centran en la solución de problemas a partir de redes de cooperación sin contar con el gobierno como una única autoridad.

Por otro lado, Jorquera, D. (2011) define la gobernanza como "un proceso de coordinación de actores, de grupos sociales, de instituciones para lograr metas definidas colectivamente en entornos fragmentados y caracterizados por la incertidumbre". Es decir que con el paso del tiempo el gobierno ha perdido autonomía y capacidad para tomar las decisiones, además la 
sociedad ha abandonado el papel de subordinación. Aguilar, F. (2010), sin embargo, para D'Eramo, D. E. (2017) la gobernanza es una respuesta a las crisis de gobernabilidad.

En cambio, para Pedro, G. Arias, J. (2013) la gobernanza provee a los actores sociales servicios públicos y establece que su papel es analizar y estudiar el rol que desempeñan los actores que no son públicos, es decir la participación de los ciudadanos en materia de decisiones políticas. Además, Coronado, O. Carreón, J. Quiroz, C. Mojica E. García, C. (2019) expresan que la gobernanza se enfoca en el desarrollo local que tiene como eje central lo social y el emprendimiento.

En este orden de ideas podemos visualizar la gobernanza como un modo que involucra diversos factores para generar desarrollo y que busca establecer una relación entre el estado y la sociedad de manera bilateral. Jorquera, D. (2011) y que por lo tanto genera reformas estatales de los enfoques de las políticas públicas. D’Eramo, D. E. (2017)

Se puede analizar que se establece una cercanía entre el emprendimiento y la gobernanza, pues como lo plantean Calvo, G. Pérez, H. Romero M. (2020) "¿cómo entender la empresa en tanto actor social y no solamente económico?" al igual que en el gobierno, la globalización tiene una fuerte influencia sobre los emprendimientos y las empresas, de acuerdo con Barón, M. (2018) la gobernanza tiene como eje central los territorios, pues su propósito es atender a las necesidades de las comunidades, por otro lado, Cantero, S. Calvo, R. (2014) señalan que el emprendimiento nace en estos espacios territoriales buscan un crecimiento social y económico. Es decir que ambas variables son generadoras de desarrollo.

Bayón, M. (2018). establece que la gobernabilidad es la que tiene la capacidad para generar políticas públicas, pero es la gobernanza la que está asociada al estado para la gestión económica. Para los autores Arteaga, K. Pereira, M. Toscano, A. Fuentes, D. Melo, M. (2019) en un estudio realizado sobre las políticas públicas para el fomento del emprendimiento en el departamento de Córdoba - Colombia, consideran que las políticas públicas pueden dar respuesta al emprendimiento y aunque se tenga un fuerte marco normativo en materia de emprendimiento, hay debilidades en el fomento de esta. 
Calvo, G. Pérez, H. Romero M. (2020) plantean que las relaciones que hay entre el territorio y el sector privado, se han ido modificando al igual que el papel de las empresas en la sociedad, es aquí cuando el territorio deja se ser visto como solamente un espacio para generar actividades económicas, pasando a ser visto como un espacio para ayudar a transformar la sociedad de manera positiva.

En este sentido Martínez L (2016). sostiene que las políticas públicas tienen como fin único modificar las conductas de los ciudadanos y que, según Coronado, O. Carreón, J. Quiroz, C. Mojica E. García, C. (2019) el emprendimiento supone el fomento de las políticas públicas para promover la productividad de los individuos.

Para Santos, J. Barroso, M. Guzmán, C. (2013). Los emprendedores son quienes están en constante búsqueda de oportunidades. Martínez, L (2018) afirma que con el paso del tiempo y con el desarrollo de los modelos de producción, los emprendimientos comenzaron a transformarse en un factor fundamental para los gobiernos. Caro $F$ (2015) ofrece una perspectiva de que los emprendedores son quienes asumen el riesgo de crear e innovar tomando como prioridad su bienestar y se puede manifestar como una estrategia para luchar en épocas de crisis.

los autores Arteaga, K. Pereira, M. Toscano, A. Fuentes, D. Melo, M. (2019) nos plantean que cuando la sociedad está atravesando diversos problemas, son las políticas públicas las que surgen para dar una respuesta, que, según Martínez, L (2018) su finalidad es enfrentar de la mano con el emprendimiento los desafíos de carácter público, pues existe la necesidad de contrarrestar el desempleo, la pobreza y otros problemas persistentes en la sociedad.

Según Martínez L (2016). Las políticas de emprendimiento se desarrollan a partir de la evidencia de un contexto en crisis donde hay una transformación de los mecanismos del gobierno y el empleo. Perdomo, G. Arias, J. (2013) plantea que los actores menos estudiados son las políticas públicas para fortalecer los emprendimientos y promover la innovación en este sentido Martínez L (2016) afirma que políticas públicas han ido ganando terreno en cuanto a su importancia para el desarrollo de la sociedad en los últimos años. 
La generación de las políticas públicas ha tenido una transformación y se han ido desplazando a los entes que no son gubernamentales, es decir que existe una participación bilateral en el diseño de las políticas públicas (Perdomo, G. Arias, J. 2013).La participación en las políticas publicas para los emprendedores se enmarca en una idea del estado como es la democracia participativa de los actores y grupos de interés para su diseño, la interacción que hay entre el estado y los ciudadanos son el reflejo de la toma de decisiones y planeación de los programas públicos. En este orden de ideas la participación de todos los actores se convierte en el eje central para formular respuestas a la sociedad y brindar nuevos enfoques, transformándose en un mecanismo para conectar las políticas con las demandas sociales. (Perdomo, G. Arias, J. Lozada N. 2013).

Caro F (2015) considera que al apoyar estas iniciativas favorecen la creación de nuevos empleos y la auto empleabilidad. Por otro lado, para Martínez L (2018) el interés por apoyar los emprendimientos por parte del gobierno cada vez es más creciente, a raíz de esto han ido surgiendo y creciendo medidas para potencializar el emprendimiento, en este sentido Perdomo, G. Arias, J. (2013) evidencia en un estudio realizado en Medellín Antioquia sobre la incubación de empresas que demuestra avances en los modelos de gobernanza sobre la gestión de políticas públicas.

Esto impulsa el desarrollo de la producción y el crecimiento económico, sin embargo, son las instituciones quienes deben favorecer los emprendimientos mediante el diseño y fomento de políticas económicas y sociales. Caro $F$ (2015) se ha ido posicionando como uno de los principales elementos que deben ser investigados, no solo desde la ciencia política o gestión pública, sino que también debe ser abordado desde diferentes disciplinas sociales. (Martínez L 2018).

Sin embargo, los autores Arteaga, K. Pereira, M. Toscano, A. Fuentes, D. Melo, M. (2019) ponen de manifiesto que el ritmo de los motores para promover el crecimiento económico del país ha tenido una desaceleración en Colombia, lo que plantea la búsqueda de otros factores que generen crecimiento económico a partir de la capacidad interna del país.

Pero tanto los emprendimientos como las políticas públicas se ven fuertemente influenciadas por las leyes que rigen el mercado, (Martínez, L 2016) en este orden de ideas Arteaga, K. Pereira, M. Toscano, A. Fuentes, D. 
Melo, M. (2019) analizan que las economías de mercado generan injusticia y afectan el bienestar de las personas.

Santos, J. Barroso, M. Guzmán, C. (2013) deja en evidencia que la desigualdad, la exclusión social y la pobreza son problemas que han ido aumentando y que con las crisis financieras como la del año 2007 en Estados Unidos y que tuvo una repercusión a nivel mundial a partir del año 2008 ha dejado en evidencia las debilidades del modelo económico y aborda la importancia de los emprendimientos para devolver a la economía mundial el altruismo y resolver los problemas a los que se enfrenta la sociedad.

Se considera que, en las últimas décadas, la economía no ha logrado dar respuesta a las necesidades para mantener una buena calidad de vida, sin embargo, algunos expertos plantean que la raíz del problema son las fallas político-institucionales lo que impide el crecimiento de la innovación y de las empresas. En este orden de ideas el emprendimiento comienza a jugar un rol importante para dar solución a los problemas a los que debe enfrentarse la sociedad aquí radica la importancia de fortalecer las políticas públicas para fomentar los emprendimientos.

Arteaga, K. Pereira, M. Toscano, A. Fuentes, D. Melo, M. (2019) analizan estas debilidades que presenta la economía pues no pueden funcionar de manera correcta sin la intervención del estado para promover las políticas públicas, sin embargo, Santos, J. Barroso, M. Guzmán, C. (2013) exponen que debido a que nos enfrentamos a un mercado que está fragmentado, las personas que asumen el rol de emprendedoras requieren de ciertas habilidades para enfrentar los desafíos que se presentan.

Para Arteaga, K. Pereira, M. Toscano, A. Fuentes, D. Melo, M. (2019) es necesario fomentar la educación y la cultura para promover el fortalecimiento de las políticas públicas y el emprendimiento y así en el largo plazo generar impactos económicos. Con esto Santos, J. Barroso, M. Guzmán, C. (2013) afirma que esto contribuye a la producción de bienes y servicios que tienen una alta demanda y al crecimiento económico para así lograr aumentar el bienestar de la población.

El emprendimiento social se posiciona como un tipo de emprendimiento que tiene como propósito luchar contra la pobreza, desigualdad social y generar pleno empleo, este según el autor es uno de los tipos que mayor desarrollo económico genera, ya que busca siempre la participación colectiva de los 
trabajadores en el capital de la empresa con el fin de tener siempre una buena calidad de vida, estos emprendimientos pretenden contribuir en la transformación de una sociedad más igualitaria económicamente. (Santos, J. Barroso, M. Guzmán, C. 2013).

Aaron, g. (2015) Expresa que los intentos en América Latina por disminuir la pobreza han sido fallidos mediante la utilización de instrumentos que han demostrado no ser los adecuados, en este orden de ideas las políticas públicas han sido diseñadas para luchar contra la pobreza, sin embargo, hay una mayor participación desde la gobernanza en el desarrollo de estas, es evidente que la política ha generado resultados, pero la economía no, Para Aguilar L (2015) es a partir de la gobernanza que nuevos actores obtengan la capacidad de buscar solución a los problemas públicos como la pobreza, los conflictos, la falta de educación y la baja esperanza de vida.

Para Arteaga, K. Pereira, M. Toscano, A. Fuentes, D. Melo, M. (2019) el esfuerzo de carácter político, social, económico y académico por fortalecer los emprendimientos en los sitios rurales de Colombia han sido mínimos para generar desarrollo económico. El emprendimiento en su rol de generar el crecimiento y desarrollo económico de un país permite aumentar el nivel de vida de las naciones.

Por esta razón, el emprendimiento es uno de los componentes que hacen parte de las acciones que se realizan desde las políticas para contribuir con el bienestar, dado a la motivación a sí mismos de los ciudadanos se convierte en un creciente deseo por crear empresas y trabajar de forma autónoma, en este sentido la autora afirma que el gobierno ha aumentado la atención a las políticas públicas de emprendimiento.

Han surgido leyes que respaldan el emprendimiento, es a partir del año 2002 cuando el SENA toma la decisión de incluir en su política el programa nacional de incubación de emprendimiento para ayudar a fortalecer este campo (Pedro, G. Arias, J. 2013) En los últimos años, las políticas públicas de emprendimiento han estado enfocadas en la generación de ingresos mediante la creación de negocios y pequeñas empresas. (Arteaga, K. Pereira, M. Toscano, A. Fuentes, D. Melo, M.2019).

La forma en la que se establecen y diseñan los programas y políticas públicas han cambiado, pues han pasado de la centralización a la descentralización del gobierno, cada vez es más creciente la participación de los ciudadanos. 
Lo que se busca con esto es fortalecer y promover la creación de emprendimientos y la competitividad de las empresas en Colombia. (Pedro, G. Arias, J. 2013).

Según Arteaga, K. Pereira, M. Toscano, A. Fuentes, D. Melo, M. (2019) en Colombia existen múltiples estudios en políticas de emprendimiento que develan la realidad del país, estas investigaciones han estado enfocadas en el entorno competitivo, lo que se busca es generar pleno empleo. Se ha venido evidenciando que los emprendedores son favorecidos por el entorno económico, esto se debe a un aumento en la demanda o por diversos factores que impone el mercado.

Aunque el emprendimiento hoy continúa siendo un fenómeno complejo de estudiar, se ha observado que las políticas públicas responden a las necesidades actuales, sin embargo, deben ser desarrolladas y fortalecidas teniendo en cuenta los diferentes mecanismos de participación ciudadana por otro lado se han formulado estrategias por parte de las universidades para promover el emprendimiento.

\section{CONCLUSIONES}

- La gobernanza es una respuesta a los problemas que enfrenta la sociedad, y esta respuesta puede ser dada mediante el uso de instrumentos como las políticas públicas, además esta propone una participación en la toma de decisiones políticas de carácter bilateral, mediante mecanismos de participación ciudadana.

- Las políticas publicas son un instrumento usado para dar respuesta a las necesidades y problemas actuales, por otro lado, debido a que la economía es volátil e incierta, las políticas tienen la capacidad para controlar en cierto nivel el comportamiento de la economía, y en caso de que se genere una crisis, esta tiene la posibilidad de contrarrestar los efectos negativos económicos.

- La economía tiene una fuerte influencia sobre el emprendimiento y viceversa, cuando la economía atraviesa por fuertes crisis es capaz de causar la quiebra de muchas empresas y acabar con los emprendimientos, por otro lado, puede aumentar el desempleo y disminuir los ingresos de las personas, sin embargo, basándose en el diseño de las políticas públicas en épocas de crisis estas son capaces de promover el autoempleo y bajar el porcentaje de la tasa de desempleo. 
- Es mediante las políticas públicas que se pueden aumentar los emprendimientos en la medida en que estas se fortalezcan, si bien en algunos casos se evidencian falencias en el marco legal para promover el emprendimiento, sin embargo, se han realizado estudios en busca de generar una mejora continua.

- El emprendimiento es catalogado como generador de crecimiento en la sociedad, este permite elevar el nivel de calidad de vida de los ciudadanos en especial si este es de carácter social, se afirma que quienes asumen el rol de emprendedores deben ser resilientes y poseer la capacidad de enfrentarse a los obstáculos que se presenten a la hora de generar la idea de negocio y de mantenerla en el mercado.

\section{REFERENCIAS}

Aaron, G. (2015) América Latina: Pobreza, Gobernanza e Investigación. Universidad de Occidente (UdeO) MÉXICO. Telos,17(3), 369 - 374. Recuperado

http://ojs.urbe.edu/index.php/telos/article/view/2259/2108

Aguilar, F. (2010). Gobernanza: El nuevo proceso de gobernar. México: Fundación Friedrich Naumann para la Libertad. Recuperado de http://repiica.iica.int/docs/B0400e/B0400e.pdf

Aguilar, L. (2015). Gobernanza y Gestión Publica. México: Fondo de Cultura Económica.

Arteaga, K. Pereira, M. Toscano, A. Fuentes, D. \& Melo, M. (2019). Políticas públicas para el fomento del emprendimiento: un análisis de las condiciones económicas del Municipio de Cotorra en el periodo 20082018. Perspectivas, (15), 42-71. Recuperado de https://revistas.uniminuto.edu/index.php/Pers/article/view/2064

Barón Colmenares, M. (2018). La gobernanza en el ordenamiento territorial local: presencias y ausencias de la participación ciudadana. Diálogos De Saberes, (48), 133-154. Recuperado de https://revistas.unilibre.edu.co/index.php/dialogos/article/view/4718/4 026

Bayón, M. (2018). El neoinstitucionalismo y el Banco Mundial: gobernabilidad y gobernanza. Economía y Desarrollo, 160(2), 12. Recuperado de http://scielo.sld.cu/scielo.php?script=sci_arttext\&pid=S0252-

$85842018000200003 \&$ Ing=es\&tlng=es.

Calvo, G., Pérez Hoyos, O. I., \& Romero Amaya, M. C. (2020). Miradas Cruzadas Sobre El Enfoque Territorial De La Responsabilidad Social, La Gobernanza Y La Sostenibilidad. OPERA - Observatorio de Políticas, 
Ejecución y Resultados de La Administración Pública, 26, 3-14. doi: https://doi-

org.bibliotecavirtual.unad.edu.co/10.18601/16578651.n26.02

Caro F. (2016) La inserción por el empleo de colectivos vulnerables. Discursos y estrategias de los agentes de inserción, en un contexto de crisis, Universidad Pública de Navarra. Departamento de Trabajo Social, Nafarroako Unibertsitate Publikoa. Gizarte Lana Saila. Recuperado de https://academica-e.unavarra.es/handle/2454/20994

Cantero, S. Calvo, R. (2014). Emprendimiento a nivel local. La necesidad de una estrategia de cooperación para el desarrollo del territorio. España: Universidad de Valencia. Recuperado de https://base.socioeco.org/docs/_jec14_comunica_a_ert_a_ert_3.pdf

Coronado, O. Carreon, J. Quiroz, C. Mojica E. \& García, C. (2019) Gobernanza del emprendimiento social responsable. Nómadas: Critical Journal of Social and Juridical Sciences, 57, 81-103.

D'Eramo, D. E. (2017). Gobernabilidad, gobernanza... en definitiva, el estado. Administración Pública Y Sociedad (APYS), (3), 126-135. Recuperado de https://revistas.unc.edu.ar/index.php/APyS/article/view/15314

Jorquera, D. (2011). Gobernanza para el Desarrollo Local. Documento de Trabajo, 6. Proyecto Conocimiento y Cambio en Pobreza Rural y Desarrollo, 1-14. Recuperado de http://www.rimisp.org/wpcontent/files_mf/1366307608n952011gobernanzaparadesarrollolocaljor quera.pdf.

Martínez, L. (2016). El discurso europeo sobre el emprendimiento. Políticas públicas, trabajo y subjetividad en el marco de la gobernanza económica europea. Universidad Pública de Navarra. Departamento de Trabajo Social, Nafarroako Unibertsitate Publikoa. Gizarte Lana Saila. Recuperado de https://academica-e.unavarra.es/handle/2454/28809

Martínez, L. (2018). Política Pública De Emprendimiento En Colombia 2006 2016: Un Análisis Cognitivo Desde Los Instrumentos De Planificación. Bogotá, Colombia: Universidad Externado De Colombia Facultad De Finanzas, Gobierno y Relaciones Internacionales.

Perdomo, G. Arias, J. (2013). Las políticas públicas de desarrollo empresarial e innovación desde la perspectiva de la nueva gestión pública y la gobernanza: caso CREAME. Apuntes del Cenes, 32(56), 175-204. Recuperado http://www.scielo.org.co/scielo.php?script=sci_arttext\&pid=S0120$30532013000200008 \&$ lng =e\&tlng=es.

Perdomo, G. Arias, J. \& Lozada, N. (2013). Análisis de las políticas públicas de desarrollo empresarial e innovación desde la perspectiva de la 
nueva gestión pública y la gobernanza: caso Barcelona activa incubadora de empresas. Perfil de Coyuntura Económica, (21),173197.

Recuperado de https://www. redalyc.org/articulo.oa?id=861/86129819009.

Santos, J. Barroso, M. \& Guzmán, C. (2013). La economía global y los emprendimientos sociales. Revista de Economía Mundial, 35, 177-196. Recuperado de https://www.redalyc.org/pdf/866/86629567010.pdf 